\title{
Effects of Prolonged Sitting Interventions on Chronic Low-Grade Inflammation in Adults: a Protocol for a Systematic Review
}

\author{
S. Azharuddin', Chythra R. Rao², B. Chandrasekaran¹, S. J Pedersen³ \\ 1 Department of Exercise and Sports Sciences, Manipal College of Health Professions, Manipal Academy of \\ Higher Education, Manipal, Karnataka, India \\ 2 Department of Community Medicine, Kasturba Medical College, Manipal Academy of Higher Education, \\ Manipal, Karnataka, India \\ 3 Active Work Laboratory, School of Education, University of Tasmania, Tasmania, Australia
}

\section{CORRESPONDING AUTHOR:}

Baskaran Chandrasekaran

Department of Exercise and Sports

Sciences

Manipal College of Health Professions

Manipal Academy of Higher Education

Marena Building

Madhav Nagar

Manipal 576104

Karnataka, India

E-mail: baskaran.c@manipal.edu

DOI:

10.32098/mltj.04.2021.02

LEVEL OF EVIDENCE: 2A

\section{ABBREVIATIONS:}

CSI: Chronic Systemic inflammation

SB: Sedentary behaviour

PA: Physical activity;

MVPA: Moderate to vigorous physical activity

LIA: Low intensity activity

CRP: C reactive protein

IL: Interleukins

IFN: Interferon

PAF: Plasminogen activating factor

IGF: Insulin like growth factors

TNF- $\alpha$ : Tumour Necrosis Factor alpha

TLF-4: Toll like factor-4

\section{SUMMARY}

Background. Chronic systemic inflammation (CSI) is linked with pathogenesis of chronic disease risk including type 2 diabetes, obesity, cardiovascular diseases and cancer. However, there is dearth of evidence to inform the stakeholders about the pooled effect of excessive sedentary behaviour or its interruptions, which may alter the CSI in adults. Our systematic review will aim to find the evidence behind the sedentary behaviour interventions on CSI. Methods. Five databases (Scopus, PubMed, Web of Science, Cochrane Central Register of Controlled Trials, Ovid Medline and CINAHL) will be searched for studies examining the influence of excessive sitting or its interruptions on CSI markers (Interleukin; C-Reactive Protein, Cytokines), its dose, gender differences and context specific settings. Studies that included healthy working, adult population will be examined by two independent reviewers.

Results. The study quality will be assessed by QualSyst tool and Cochrane Risk of Bias tools using Revman 5.4. The mean effect size of the sitting interventions on CSI markers will be presented after exploring for potential publication bias. Appropriate visualisation of the effects of the outcome measures of interest will be assessed through Forrest plots to assess the direction, consistency and size of the intervention. Conclusions. Potential associations between excessive sitting and the effects of interruption interventions on CSI will be explored after assessing the quality of the studies.

\section{KEY WORDS}

Chronic systemic inflammation; sedentary behaviour; interleukin; C-reactive protein; cytokines; prolonged sitting; interrupted sitting. 


\section{OPERATIONAL DEFINITIONS: adapted from (1)}

Metabolic equivalent (MET)s. Physiological measure of energy expenditure expressing the intensity of physical activities. One MET is expressed as energy expenditure at rest $\approx 3.5 \mathrm{ml} / \mathrm{kg} / \mathrm{min}$.

Sedentary behaviour (SB). Any waking behaviour (sitting, lying, reclining) that is characterised by low energy expenditure ( $<1.5 \mathrm{METs})$.

Moderate to vigorous physical activity (MVPA). Any bodily movement or exercise that increases energy expenditure by $>3$ METs and exertion level of 5-6 on a scale of 10 .

\section{INTRODUCTION}

Chronic low-grade inflammation attributed to sedentary behaviour (SB) can have deleterious effects on work, quality of life, and in severe cases eventuate into a metabolic syndrome. Chronic systemic inflammation (CSI) is associated with the incidence of a myriad of chronic diseases including cardiovascular diseases, type 2 diabetes, fatty liver diseases, cancer, musculoskeletal disorders, depression, dementia and Alzheimer's disease (2). Persistent circulating markers such as proinflammatory cytokines [interleukins (IL-6, IL-8), tumour necrosis factor (TNF- $\alpha$ )], acute phase proteins [C-reactive protein $(\mathrm{CRP})$ and plasminogen activating factor $(\mathrm{PAF})]$ may perpetuate underlying chronic disease risk via possible mechanisms such as high postprandial hyperglycaemia, insulin resistance, oxidation stress, adipose tissue dysregulation, neurodegeneration, triglyceridemia and atherosclerosis $(2,3)$. Hence, these immunomodulatory pathways become the potential target for SB interventions that increase daily physical activity $(\mathrm{PA})$ to reduce disease risk and morbidity common in clinical populations.

\section{Anti-inflammatory effects of PA or SB interventions}

The anti-inflammatory effects of PA are evident in epidemiological studies that report low CSI in primitive, non-industrialised populations such as Hadza of Tanzania, Shuar of Amazon and the Tsimane foragers of Bolivia $(4,5)$. Hence, adding $\mathrm{PA}$ to reduce $\mathrm{SB}$, in any form or volume, may aid in reducing the cardiometabolic risk associated with CSI in the mechanised world.

Large epidemiological studies have consistently shown that high SB and low levels of PA lead to visceral adiposity and affect inflammatory mediators (listed above) adversely leading to chronic disease $(5,6)$. Though limited and inconsistent, early empirical evidence claims that moderate to vigorous PA (MVPA) such as aerobic exercise training could help in reducing CSI and resulting cardiometabolic risk in contemporary men (7). Recent systematic reviews in this
Light Intensity physical activity (LIA). Physical activity performed at an intensity between 1.5 and 3 METS at one's personal capacity and exertion level of 2-4 on a scale of 10 .

Physical inactivity. Insufficient physical activity i.e., non-compliant with the global activity recommendations of at least 150 minutes of MVPA per week.

Microbreaks. Transient short breaks during the typical workday not lasting for more than 2-3 minutes.

area have reported a moderate reduction of IL- 6 , TNF- $\alpha$ and CRP with leisure time MVPA $(8,9)$. Skeletal muscle contraction, $\beta$-oxidation, increased sensitivity of adipose tissue to epinephrine associated lipolysis and increased oxidation of intramyocellular triglycerides are proposed to reduce inflammation through toll like receptor (TLF4) activation, limiting adipose expansion and limiting proinflammatory signalling activation (10). Nonetheless, the uptake of recommended weekly levels of MVPA in contemporary men is less than optimal.

\section{Emerging evidence of light activities on inflammation}

While only a small amount of waking hours is spent in MVPA (0.58 hrs/day), more of the day is spent in light intensity activities (LIA) that include standing or stepping $(5.19 \mathrm{hrs} /$ day). Most of the day is spent in SB (9.53 hrs/day) including prolonged bouts of sitting $(11,12)$ (figure 1). As standing or stepping is more ubiquitous than the MVPA, LIA has become an appealing intervention target for reducing CSI $(12,13)$. Many consider prolonged sitting an independent disease risk factor from general SB, making the workplace a primary target for lifestyle behavioural interventions $(6,14)$. Nonetheless, the anti-inflammatory effects of such lifestyle interventions have yet to be systematically reviewed.

For a decade now, sizable experimental trials have utilised SB interventions or LIA to interrupt or replace prolonged sitting to investigate cardiometabolic risk factors (15) such as postprandial hyperglycaemia (16), triglyceridemia (17), blood pressure (18) and anthropometric measures such as waist circumference and body mass index $(19,20)$. Despite evidence suggesting that interrupting or replacing sitting with LIA, both acutely ( $<7$ days) (6) and chronically (> 2 weeks) (19), can have a moderate reduction in cardiometabolic risk factors. Nonetheless, inconsistencies in the reporting of CSI markers remain. For example, Henson and colleagues (2018) found that iso-temporal substitution of sitting with 60 mins of stepping yielded better reduction (- 


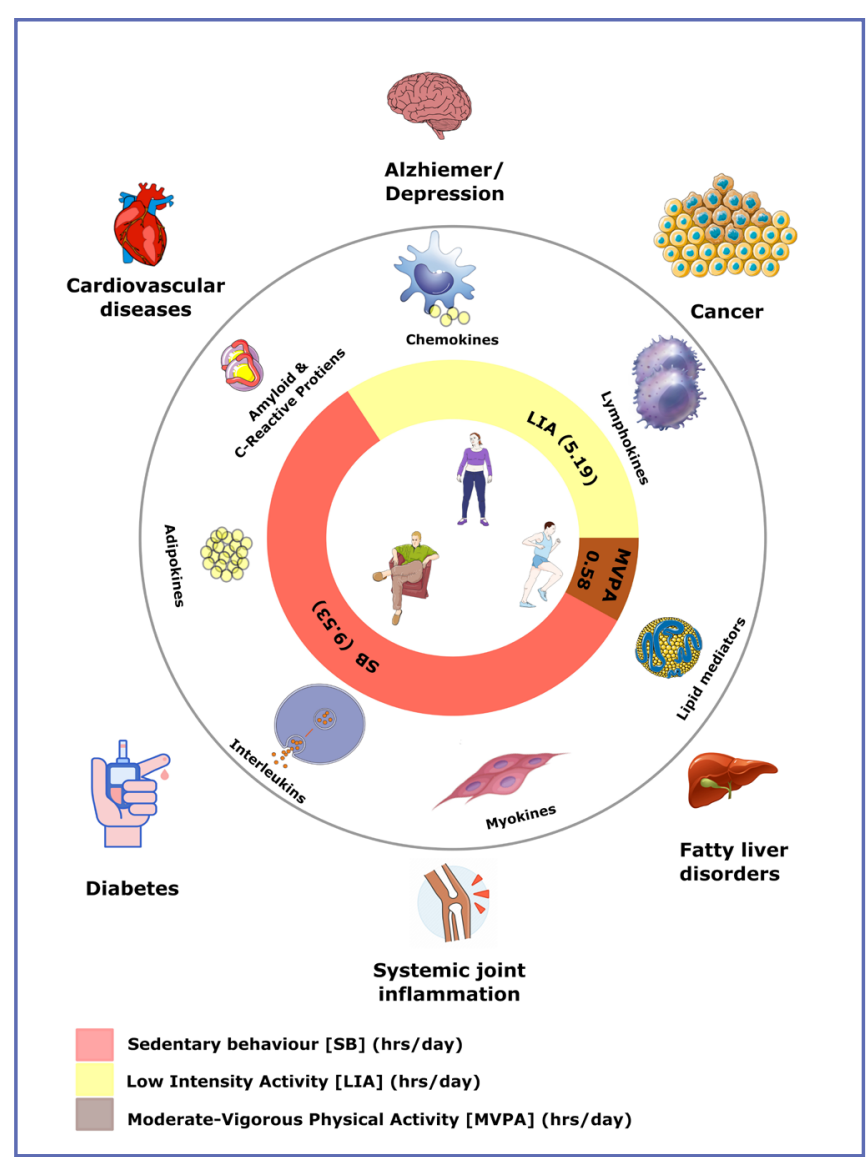

Figure 1. Association of daily wake hour activity, chronic inflammation and chronic diseases. (central circle representing daily physical activity is drawn from the data source) (11).

28\%) in IL-6 compared to standing (- 5\%). Regular sitting breaks also reduced IL- 8 by $0.19 \mathrm{pg} / \mu \mathrm{g}$, whereas uninterrupted sitting increased IL- 8 by 0.31 after a four hour trial period (21). These findings warrant further investigation.

\section{Problem statement}

While substantial evidence exists to claim the chronic disease risk due to CSI and scheduled exercise or MVPA on inflammation, the evidence regarding altered inflammatory physiology with SB and LIA is still emerging (22) (figure 2). Hence there is a need to establish a systematic review to pool the inflammatory effects of SB and LIA to inform the policy makers for better SB or PA interventions in community and context specific settings for combating inflammation and resulting chronic disease risk. This problem statement is illustrated in figure 2 .

This review will aim to consolidate the existing evidence to determine the effects of both uninterrupted and interrupted

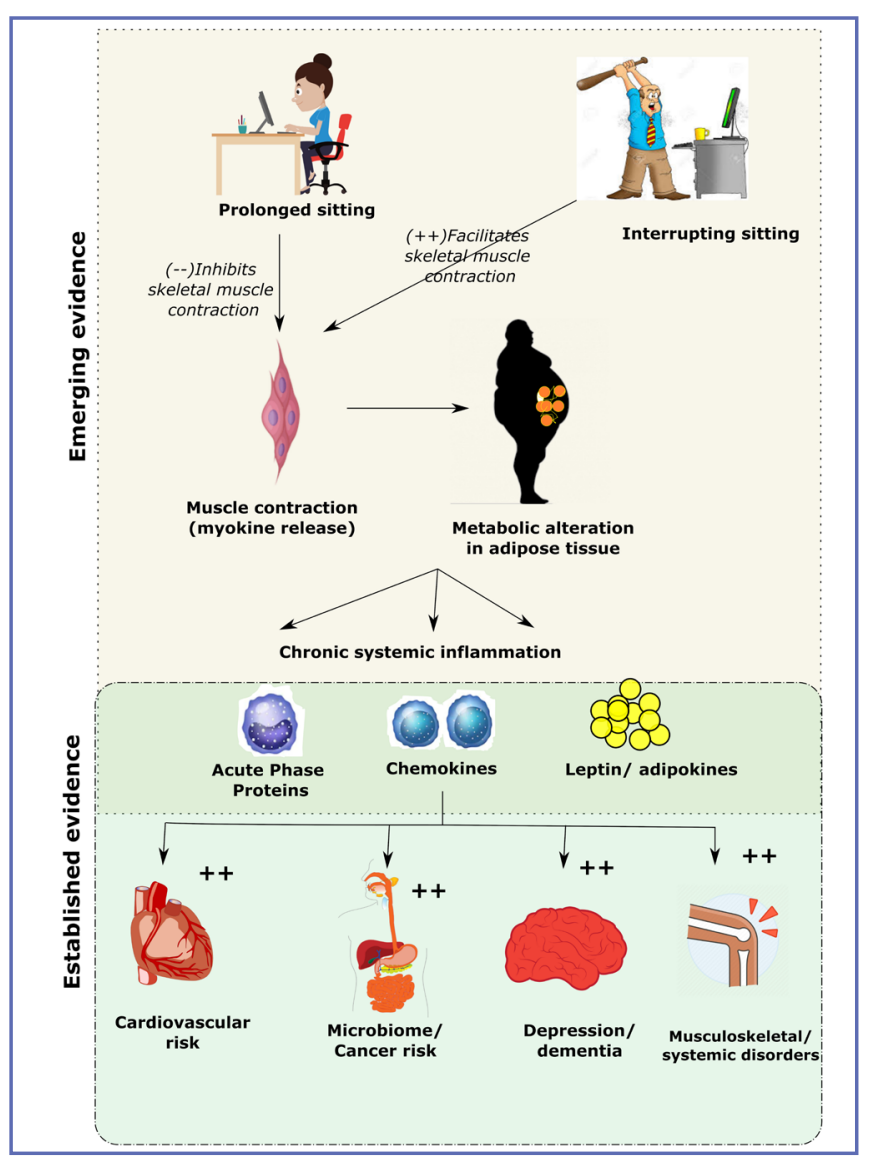

Figure 2. Graphical summary of the problem statement presented in the systematic review.

sitting on systemic inflammatory markers (proinflammatory cytokines and acute phase proteins). We aim to determine if uninterrupted sitting is associated with increased proinflammatory cytokines [IL-1, TNF, adipokines, tissue plasminogen activator inhibitor (t-PAI)], chemokines (IL-8) and acute phase proteins in contemporary men, and negatively associated with IL-6 and CRP. Further, we will investigate whether and to what extent do sitting interruptions alter the proinflammatory cytokines (IL-1, IL-6, CRP, TNF, adipokines, t-PAI), chemokines (IL-8) and acute phase proteins in adults.

\section{METHODS}

\section{Reporting methods and registration}

The methodology of the present systematic review protocol is reported based on the guidelines of the Preferred Reporting Items for systematic reviews and Meta-Analyses Protocol (PRISMA-P, 2015). A completed copy of PRISMA-P checklist 
is provided as appendix $\mathbf{1}$. The review protocol is prospectively registered in the International Prospective Register of Systematic Reviews (CRD42020216611; www.crd.york.ac.uk/PROSPERO). The research review was conducted ethically according to international standards and as required by the journal as described in Padulo et al. (2018) (23).

\section{Data sources and search criteria}

A comprehensive search of peer reviewed electronic databases (Scopus, Web of Science, Ovid Medline, Embase, PubMed Medline, Cochrane Central Register of Controlled Studies, Scientific Electronic Library Online, Cumulative Index to Nursing and Allied Health Literature) will be performed by the primary author with the assistance of librarian from inception until May 15 $5^{\text {th }}, 2020$. Besides trial registries including International Trials Registry Platform (https://www.who.int/clinical-trials-registry-platform), meta Register of Controlled Trials (http://www.isrctn. com/page/mrct) and Clinical Trials.gov (https://www.clinicaltrials.gov/) will be checked for relevant registered trials and published studies for possible inclusion to the review. Further non peer reviewed databases such as OpenGrey, Google scholar will also be searched. The Medical Subject Heading $(\mathrm{MeSH})$ terms and appropriate combinations will be identified for problem (SB), intervention (interrupt or replace), comparison (prolonged sitting or sedentary position) and outcome (inflammatory markers as exploded from $\mathrm{MeSH}$ browser of US National Library of Medicine) with appropriate qualifiers and $\mathrm{MeSH}$ tree structures. Appropriate combination of the keywords to identify the article that investigated prolonged sitting or interrupting prolonged sitting on inflammatory markers in healthy adult participants will be utilised. Appropriate wildcards not limiting to *, ?, / and proximity search using "N/n, adj n, Pre/n will be used appropriately for retrieving larger searches. For example, search terms such as "behavio?r", "sedentar*", "activit*", "inflammat*" will be framed as appropriate to the PICO search mentioned below. The reference list of the articles meeting the inclusion criteria including earlier systematic reviews will also be scanned for possible additional eligible studies. Example search strategy for Scopus, Ovid Medline and CINAHL are provided as appendix 2.

\section{Eligibility criteria}

The study question, search, extraction of the studies will be guided by the Population, Intervention or Exposure, Comparison, Outcome and Study (PICOS) design criteria to be used as the 'yardstick' for study eligibility.

\section{Population}

Studies which included apparently healthy adults $(\geq 18$ years; non-smokers; not taken any anti-inflammatory medications) will be added to the review. Studies which conducted experiments on healthy adults who worked full time or part time in desk-based jobs will be included.

\section{Intervention}

We will include any studies that aimed to reduce SB or increase PA in isolation or in combination of various intervention strategies as outlined in table I. Studies which have

Table I. Strategy categories and workplace intervention.

\begin{tabular}{ll}
\hline $\begin{array}{l}\text { Strategy categories for replacing or interrupting } \\
\text { occupational sitting }\end{array}$ & Interventional activities \\
\hline Restructuring physical environment & $\begin{array}{l}\text { Active workstations such as sit-stand, treadmill, biking desks; adding gym } \\
\text { facilities, bike parking spaces. }\end{array}$ \\
\hline Changing organisation culture and norms & $\begin{array}{l}\text { Office environment that supports schedule breaks, standing and walking } \\
\text { meetings, stair use, lunch walks and games, annual sports meet. }\end{array}$ \\
\hline Information and counselling & $\begin{array}{l}\text { Provide group or individual counselling strategies like goal setting, strategies } \\
\text { based on self-determination and health belief models like dangers of sitting, } \\
\text { benefits of move more at work, details of online information (websites, online/ } \\
\text { text messaging). }\end{array}$ \\
\hline Prompts/cues & $\begin{array}{l}\text { Computer based, sensor-based goal setting prompts for promoting walk and } \\
\text { office-based activities. }\end{array}$ \\
\hline Material reward/incentivization & $\begin{array}{l}\text { Pedometer step based; stair climb challenges. } \\
\text { Monitoring of outcome }\end{array}$ \\
\hline Activity logs and monitoring workplace SB. \\
\hline
\end{tabular}


administered SB interventions for acute periods (at least 1 hour to $\leq 7$ days) or chronic periods ( $\geq 7$ days) will be included. Studies that have administered any form of the intervention intended to interrupt or replace the sitting period (walk, stand, calisthenics, resistance exercises, treadmill walk, stair climb time, steps, exercise, dance) will be grouped into strategy categories and interventions will be mapped with the behaviour change techniques as mentioned in table $\mathbf{I}$.

\section{Comparator}

The studies that investigated occupational SB within groups and compared with parallel groups that received other treatments, comparison for a specified time, or without exposure of sitting interventions, or any form of intervention meant to interrupt occupational SB will be included. Since workplace interventions are often administered in groups, control groups may be a usual work group (often perceived to continue their routine work or received standard information for workplace wellbeing).

\section{Outcome measures}

Studies that have measured explicitly any of the following outcome measures in an isolated form or combined forms: adipokines (leptin, adiponectin), pro inflammatory cytokines (IL-6, IL-8, TNF- $\alpha$ ), acute phase proteins including CRP, leptin, t-PAI and insulin like growth factor. Studies should report odds ratio in cases of exposure, mean differences or effect sizes in cases of interventional studies.

\section{Study design}

We will consider any type of study (experimental, non-experimental observational studies) that explored association between SB and any of the above-mentioned inflammatory markers. Thus, included studies will be randomised, non-randomised, cluster randomised trials, single group before-after studies, repeated measures or interrupted time series as defined by Cochrane's Effective Practise and Organisation of Care taxonomy (24).

\section{Nature of publication}

The potential studies to be included should be published in English and involved humans. The publications will not be limited to context specific settings as laboratory settings mimicking contextual settings have been explored. Conference proceedings, abstracts, editorials, case reports will be excluded.

\section{Article selection}

All the retrieved study references will be imported into a collaborative systematic review software, "Rayyan web application" (Qatar Computing Research Institute, Qatar, https://rayyan.qcri.org/welcome). After the removal of duplicates, two authors (AS, BC) will independently screen the titles and abstracts of the retrieved articles from the systematic search to include potentially relevant studies using PICO as proposed earlier. If the potential studies are labelled as 'included' or 'may be' by both the reviewers (AS and BC), full text articles will be downloaded and screened for eligibility based on the inclusion criteria mentioned above. All the possible reasons for exclusion of the studies will be documented. In case of any missing data in the included studies, the authors will be contacted for necessary information. Any discrepancies will be resolved through mutual agreement between both the authors and if not resolved, the third reviewer (SP) will be consulted. The results of the selection process with the included and excluded studies and possible reasons for exclusion will be illustrated using Preferred Reporting Items for Systematic Reviews and Meta-Analyses (PRISMA) flow diagram. A completed PRISMA checklist will be provided at the final reporting.

\section{Data extraction and quality check}

A customised data extraction sheet will be used to extract the following details:

1. author, year, country of the study origin, design of the study, setting (location, environment);

2. duplicate study, study protocol publication or registration number provided;

3. participant characteristics (gender, number contacted, allocated intervention, completed the intervention, lost to follow-up, age, job nature, inclusion/ exclusion criteria), sedentary behaviour (measurement technique, time of measurement);

4. intervention (break time, frequency, intensity, duration, type of break, washout between the breaks, follow-up);

5. behavioral change technique embeded in the intervention and theoretical model underpinning the behavioral change technique;

6. outcome measures (type of the inflammatory marker, method of measurement, unit of the measure, validity and reliability, blinding, mean change or association after the intervention or exposure);

7. intervention effects (effect size, hazard or risk ratio, $95 \%$ confidence interval, standard deviation), intention to treat or per-protocol analysis, acknowledged limitations, possible bias (selection, reporting), conclusions and funding or any other source of conflicts of interest. 
The primary investigator will extract data from the articles and the other two investigators (BC and CRR) will verify the extracted data in the customised, a priori data extraction sheets. First ten articles will be extracted to the data sheet independently by the three reviewers (AS, BC, CRR) as a pilot test. Effect sizes and the estimates of CSI markers (acute phase proteins, chemokines, interleukins, cytokines, t-PAI, CRP, TNF) will be calculated from the observational and randomised trials according to Higgins and colleagues (2020). In case of any incomplete or missing data in the included studies, the corresponding author of the included studies will be contacted by email to retrieve appropriate information.

The quality assessment tool, QualSyst (Alberta Heritage Foundation, Canada) for qualitative studies will be used to assess the quality of the behavioural interventions and observational studies (25). The QualSyst tool for qualitative studies includes 10 questions for which the reviewer will be scoring 'yes' (2), 'partial' (1) and 'no' (0) for each question on the checklist. Items not applicable to the 10 questions will be excluded from the calculation of summary score. A summary score for each study is determined by adding the number of "yes" scores $x 2$ plus the number of "partial" scores $\mathrm{x} 1$ then dividing by 20 (the maximum number) minus the number of "not applicable" scores x2 (26). Thus, the quality of the study can be illustrated as: strong (summary score of $>0.80$ ), good (summary score of 0.71-0.79), adequate (summary score of $0.50-0.70$ ) and limited (summary score of $<0.50)(27)$.

Although appropriate for evaluating the quality of the study, QualSyst has been suggested to lack the ability to detect the biases present within a study (27). Hence, we will also assess the risk of bias present within studies using the Cochrane Risk of Bias tools (28) as elaborated below.

\section{Risk of bias}

After the quality check, the studies will be examined for apparent risks of publication bias including imprecision, inconsistency and indirectness. Risk of bias will be assessed using the Cochrane Risk of Bias tools (ROB-2 for RCTs; 2016 and ROBINS-I tool for non-randomised studies; 2016) (28). Bias (selection, attrition, detection, reporting) will be assessed using seven domains: 1) random sequence generation, 2) allocation concealment, 3) deviations from intended interventions, 4) missing outcome data, 5) selective outcome reporting, and 6) blinding of participants; 7) blinding of outcome assessors as reported in Cochrane Handbook for systematic reviews, Section 7.6 (29). Based on the criteria of the signalling questions as outlined by the Higgins et al., 2020 (29), the primary investigator (AS) will rate the risk of bias in each domain as 'low', 'unclear' or 'high' along with the justification of the reviewer's decision on the excluded study.

\section{Sub-group analysis}

Subgroup analysis will be performed to further understand the dose-response relationship of the frequency of prolonged sitting interruptions and the resultant inflammatory effects. We will consider intervention type $(19,30)$, intensity of the movement breaks $(30,31)$ and any differences attributed to gender (32). Further, our investigation will also include studies designed for working adults with specific conditions including hypertension, diabetes, arthritis, obesity and cardiovascular diseases. Other variables of interest will include: region of origin (US, Australia, European countries), age criteria (young adults: 19 to 35 vs older adults: > 35 years), follow-up duration (weeks, months, years), sitting assessment (objective $v s$ self-reported) and the effect of above variables on the inflammatory markers will be individually explored.

\section{Data analysis}

Meta-analyses will be performed if two or more studies are homogenous in population, methodology (micro breaks, breaks administration), outcome measurements and reporting. The mean and standard deviations of the inflammatory markers $(\mathrm{mg} / \mathrm{dL}, \mathrm{pg} / \mathrm{dL})$ during the pre-post trial periods or mean differences with their standard deviations of both intervention and comparator groups will be entered into the Revman 5.4.1 (The Nordic Cochrane Centre, Copenhagen, Denmark). If meta-analyses are not possible, the study findings such as characteristics, sitting time (h/day), dosage of intervention (break mode, time and the duration), comparison of methods in assessing inflammatory markers, quality of the data presented, magnitude of change reported, bias, intention to treat analysis or per-protocol analysis will be presented as qualitative narrative syntheses.

The quality of evidence for each outcome of interest will be assessed using Grading of Recommendations, Assessment, Development and Evaluation (GRADE) levels of evidence as 'very low', 'low', 'moderate', and 'high'. The quality of evidence will be imported from the Cochrane workspace into GRADE Profiler version 3.6 (GRADEpro working group, McMaster University, ON, Canada) for rating the quality of evidence and the final evidence summary table will be created.

The statistical heterogeneity across the included studies will be assessed using tests of Chi square $\left(\chi^{2}\right)$ and homogeneity $\left(\mathrm{I}^{2}\right)$ with $\mathrm{I}^{2}<50$ indicates a low risk of homogeneity whereas $\mathrm{I}^{2}$ $>50$ indicates a high risk of homogeneity. We will use a fixed 
effects model if $\mathrm{I}^{2}<50 \%$ and random effects modelling if $\mathrm{I}^{2}>$ $50 \%$ using an inverse variance method. DerSimonian-Laird method calculates the random effects by measuring standard errors of the adjusted study estimates as outlined in Cochrane Handbook for systematic reviews, section 9.4.3.1 (29). Pooled effects of the risk ratios will be calculated for observational studies enquiring the risk of sitting time with chronic low-grade inflammation. For intervention studies (to answer the second question), pooled mean differences effects will be estimated from the individual intervention effects (mean differences within and between the groups). Effect sizes will be calculated as standardized mean differences, where $<0.2$ was defined as trivial, 0.2 to 0.4 as small, 0.4 to 0.7 as moderate, and $>0.7$ as large (29).

Leave-one-out sensitivity analyses will be performed by excluding one trial at a time to test the robustness of the pooled results and to prevent conclusions from being too dependent on an individual study (Cochrane Handbook for systematic reviews, Section 10 (29)). Possible publication bias will be visually analysed by contour enhanced funnel plots as guided by Begg's rank correlation test (33). If the Beggs test is underpowered, we will use non-parametric Tweddie's trim and fill method to calculate bias-corrected estimates (33). If enough homogenous data (method of intervention, outcome measure) is available, meta-analyses and forest plots will be created by Revman 5.4.1 to compute the pooled mean differences or the effect size of the intervention effect of interrupting sitting on each of the inflammatory markers.

\section{DISCUSSION}

To our knowledge, the present systematic review will be the first to examine the effectiveness of SB interventions specifically on chronic low-grade inflammation in context specific and laboratory settings of sedentary workplaces. There is a need in pooling the effects of SB and its interruptions on CSI which could facilitate human resources policy development to reduce sitting time for ameliorating the chronic disease risk. This systematic review will provide a rigorous examination of SB interventions on the CSI while considering the challenges and limitations associated with measuring inflammatory markers in a context specific workplace setting.

\section{Potential limitations}

The SB interventions may vary across different settings and countries using variety of behaviour change compo- nents; hence we anticipate a high heterogeneity in the potential studies that may be included in our review (19). Unpublished studies will not be included in the present review which may bias the results and may potentially limit the generalisation of the review results. Nevertheless, Hawthorne effects of the control group (where the participants may increase in the outcome of interest due to increased awareness of being observed over the trial period) may be inevitable in the studies as implemented in organisation not in 'real world setting'. Further we could probably expect only modest effect size between control and intervention groups due to the social desirability bias (participants of potential studies with tendency to report societal norms) and effect of measurement (high biomarker in control group causing behaviour change in absence of intervention). Further mixing of subjective and objective measure of $\mathrm{SB}$ assessment at context specific setting may affect the results of the review generalisation, however we are planning to conduct a different subgroup analysis for the subjective and objective SB measurements in the included studies.

\section{HUMAN RIGHTS}

All procedures performed in studies involving human participants were in accordance with the ethical standards of the institutional and/or national research committee and with the 1964 Helsinki declaration and its later amendments or comparable ethical standards.

\section{CONTRIBUTIONS}

SP, CRR \& BC conceived and designed the research, AS \& BC framed the protocol for the systematic review. $\mathrm{BC}$ drafted the manuscript. SP and CRR proofread the manuscript.

\section{ACKNOWLEDGEMENTS}

The authors wish to thank Dr Fiddy Davis PhD, Head of the Department, Department of Exercise and Sports Sciences, Manipal Academy of Higher Education, Manipal, Karnata$\mathrm{ka}$, India for his continuous support and motivation for the research and manuscript.

\section{CONFLICT OF INTERESTS}

The authors declare that they have no conflict of interests. 


\section{REFERENCES}

1. Bull FC, Al-Ansari SS, Biddle S, et al. World Health Organization 2020 guidelines on physical activity and sedentary behaviour. Br J Sports Med 2020;54(24):1451-62.

2. Furman D, Campisi J, Verdin E, Carrera-Bastos P, Targ S, Franceschi $\mathrm{C}$, et al. Chronic inflammation in the etiology of disease across the life span. Nat Med 2019;25(12):1822-32.

3. Klop B, Proctor SD, Mamo JC, Botham KM, Castro Cabezas $\mathrm{M}$. Understanding postprandial inflammation and its relationship to lifestyle behaviour and metabolic diseases. Int J Vasc Med 2012;2012:947417.

4. Gurven M, Jaeggi AV, Kaplan H, Cummings D. Physical activity and modernization among Bolivian Amerindians. PLoS One 2013;8(1):e55679.

5. Raichlen DA, Pontzer H, Harris JA, et al. Physical activity patterns and biomarkers of cardiovascular disease risk in hunter-gatherers. Am J Hum Biol 2017;29(2).

6. Saunders TJ, Larouche R, Colley RC, Tremblay MS. Acute sedentary behaviour and markers of cardiometabolic risk: a systematic review of intervention studies. J Nutr Metab 2012;2012:712435.

7. Tulasiram B, Chandrasekaran B. Are Smartphones Better in Guiding Physical Activity Among Sedentary Young Adults? A Randomised Controlled Trial. Muscles Ligaments Tendons J 2021;11(1):83-91.

8. Zheng G, Qiu P, Xia R, et al. Effect of Aerobic Exercise on Inflammatory Markers in Healthy Middle-Aged and Older Adults: A Systematic Review and Meta-Analysis of Randomized Controlled Trials. Front Aging Neurosci 2019;11:98.

9. Rose GL, Skinner TL, Mielke GI, Schaumberg MA. The effect of exercise intensity on chronic inflammation: A systematic review and meta-analysis. J Sci Med Sport 2020.

10. Burini RC, Anderson E, Durstine JL, Carson JA. Inflammation, physical activity, and chronic disease: An evolutionary perspective. Sports Med Health Sci 2020;2(1):1-6.

11. Luis de Moraes Ferrari G, Kovalskys I, et al. Association of moderate-to-vigorous physical activity with neck circumference in eight Latin American countries. BMC Public Health 2019;19(1):809.

12. Henson J, Edwardson CL, Bodicoat DH, et al. Reallocating sitting time to standing or stepping through isotemporal analysis: associations with markers of chronic low-grade inflammation. J Sports Sci 2018;36(14):1586-93.

13. Henson J, Yates T, Edwardson CL, et al. Sedentary time and markers of chronic low-grade inflammation in a high risk population. PLoS One. 2013;8(10):e78350.

14. Saunders TJ, Atkinson HF, Burr J, MacEwen B, Skeaff CM, Peddie MC. The Acute Metabolic and Vascular Impact of Interrupting Prolonged Sitting: A Systematic Review and Meta-Analysis. Sports Med 2018;48(10):2347-66.

15. Zongpa TC, Chandrasekaran B, Arumugam A. Effectiveness of A Smartphone Directed Physical Activity Program on Cardiometabolic Disease Risk in Desk-Based Office Employees - A Pragmatic, Two-Arm, Parallel, Cluster Randomised Trial. Muscles Ligaments Tendons J 2021;10(4):713-23.

16. Duvivier BMFM, Schaper NC, Koster A, et al. Benefits of Substituting Sitting with Standing and Walking in Free-Living Conditions for Cardiometabolic Risk Markers, Cognition and Mood in Overweight Adults. Front Physiol 2017;8:353.

17. Loh R, Stamatakis E, Folkerts D, Allgrove JE, Moir HJ. Effects of Interrupting Prolonged Sitting with Physical Activity Breaks on
Blood Glucose, Insulin and Triacylglycerol Measures: A Systematic Review and Meta-analysis. Sports Med 2020;50(2):295-330.

18. Mainsbridge C, Ahuja K, Williams A, Bird ML, Cooley D, Pedersen SJ. Blood Pressure Response to Interrupting Workplace Sitting Time With Non-Exercise Physical Activity Results of a 12-Month Cohort Study. J Occup Environ Med 2018;60(9):769-74.

19. Hadgraft NT, Winkler E, Climie RE, et al. Effects of sedentary behaviour interventions on biomarkers of cardiometabolic risk in adults: systematic review with meta-analyses. Br J Sports Med 2021;55(3):144-54.

20. Júdice PB, Silva AM, Santos DA, Baptista F, Sardinha LB. Associations of breaks in sedentary time with abdominal obesity in Portuguese older adults. Age (Dordr) 2015;37(2):23.

21. Dogra S, Wolf M, Jeffrey MP, et al. Disrupting prolonged sitting reduces IL-8 and lower leg swell in active young adults. BMC Sports Sci Med Rehabil 2019;11:23.

22. Chandrasekaran B, Pesola AJ, Rao CR, Arumugam A. Does breaking up prolonged sitting improve cognitive functions in sedentary adults? A mapping review and hypothesis formulation on the potential physiological mechanisms. BMC Musculoskel Dis 2021;22(1):274.

23. Padulo J, Oliva F, Frizziero A, Maffulli N. Basic principles and recommendations in clinical and field science research: 2018 update. Muscles Ligaments Tendons J 2018;8(3):305-7.

24. Mazza D, Bairstow P, Buchan $\mathrm{H}$, et al. Refining a taxonomy for guideline implementation: results of an exercise in abstract classification. Implement Sci 2013;8:32.

25. Chastin SFM, De Craemer M, De Cocker K, et al. How does light-intensity physical activity associate with adult cardiometabolic health and mortality? Systematic review with meta-analysis of experimental and observational studies. Br J Sports Med 2019:53(6):370-6.

26. Kmet L, Lee R, Cook L. Standard Quality Assessment Criteria For Evaluating Primary Research Papers from a variety of fields: Alberta Heritage Foundation for Medical Research 2004.

27. Maharaj S, Harding R. The needs, models of care, interventions and outcomes of palliative care in the Caribbean: a systematic review of the evidence. BMC Palliat Care 2016;15:9.

28. Schünemann HJ, Cuello C, Akl EA, et al. GRADE guidelines: 18. How ROBINS-I and other tools to assess risk of bias in nonrandomized studies should be used to rate the certainty of a body of evidence. J Clin Epidemiol 2019;111:105-14.

29. Higgins JPT, Thomas J, Chandler J, et al. Cochrane Handbook for Systematic Reviews of Interventions: Cochrane 2020. Available at www.training.cochrane.org/handbook.

30. Loh R, Stamatakis E, Folkerts D, Allgrove JE, Moir HJ. Effects of Interrupting Prolonged Sitting with Physical Activity Breaks on Blood Glucose, Insulin and Triacylglycerol Measures: A Systematic Review and Meta-analysis. Sports Med 2020;50(2):295-330.

31. Chrismas BCR, Taylor L, Cherif A, Sayegh S, Bailey DP. Breaking up prolonged sitting with moderate-intensity walking improves attention and executive function in Qatari females. PLoS One 2019;14(7):e0219565.

32. Toomingas A, Forsman M, Mathiassen SE, Heiden M, Nilsson T. Variation between seated and standing/walking postures among male and female call centre operators. Bmc Public Health 2012;12.

33. Lin L, Chu H. Quantifying publication bias in meta-analysis. Biometrics 2018;74(3):785-94. 


\section{SUPPLEMENTS}

Appendix 1. PRISMA-P (Preferred Reporting Items for Systematic review and Meta-Analysis Protocols) 2015 checklist: recommended items to address in a systematic review protocol*.

\begin{tabular}{|c|c|c|c|}
\hline $\begin{array}{l}\text { Section and } \\
\text { topic }\end{array}$ & $\begin{array}{l}\text { Item } \\
\text { No }\end{array}$ & Checklist item & Reported page \\
\hline \multicolumn{4}{|c|}{ ADMINISTRATIVE INFORMATION } \\
\hline \multicolumn{4}{|l|}{ Title: } \\
\hline $\begin{array}{l}\text { Identifica- } \\
\text { tion }\end{array}$ & 1a & $\begin{array}{l}\text { Identify the report as a protocol of a sys- } \\
\text { tematic review }\end{array}$ & 1 \\
\hline Update & $1 b$ & $\begin{array}{l}\text { If the protocol is for an update of a previ- } \\
\text { ous systematic review, identify as such }\end{array}$ & NA \\
\hline Registration & 2 & $\begin{array}{l}\text { If registered, provide the name of the regis- } \\
\text { try (such as PROSPERO) and registration } \\
\text { number }\end{array}$ & 6 \\
\hline
\end{tabular}

Authors:

Contact 3a Provide name, institutional affiliation, e-mail address of all protocol authors; provide physical mailing address of corre-

Title Page sponding author

Contribu- $3 b$ Describe contributions of protocol authors tions and identify the guarantor of the review

Title Page

\begin{tabular}{lll}
\hline Amendments $\quad 4 \quad \begin{array}{l}\text { If the protocol represents an amendment of } \\
\text { a previously completed or published proto- } \\
\text { col, identify as such and list changes; other- } \\
\text { wise, state plan for documenting important } \\
\text { protocol amendments }\end{array}$ & NA \\
\hline
\end{tabular}

Support:

Sources $\quad 5 \mathrm{a}$ Indicate sources of financial or other support for the review

Title Page

Sponsor $5 b \quad$ Provide name for the review funder and/or sponsor

NA

Role of $\quad 5 \mathrm{c} \quad$ Describe roles of funder(s), sponsor(s), sponsor or and/or institution(s), if any, in developing funder the protocol

\begin{tabular}{llll}
\hline INTRODUCTION & & \\
\hline Rationale & 6 & $\begin{array}{l}\text { Describe the rationale for the review in the } \\
\text { context of what is already known }\end{array}$ & $3 \& 4$ \\
\hline Objectives & 7 & $\begin{array}{l}\text { Provide an explicit statement of the ques- } \\
\text { tion(s) the review will address with refer- } \\
\text { ence to participants, interventions, compar- } \\
\text { ators, and outcomes (PICO) }\end{array}$ & 5 \\
\hline METHODS & &
\end{tabular}




\begin{tabular}{|c|c|c|c|}
\hline $\begin{array}{l}\text { Eligibility cri- } \\
\text { teria }\end{array}$ & 8 & $\begin{array}{l}\text { Specify the study characteristics (such as } \\
\text { PICO, study design, setting, time frame) } \\
\text { and report characteristics (such as years } \\
\text { considered, language, publication status) } \\
\text { to be used as criteria for eligibility for the } \\
\text { review }\end{array}$ & $7-9$ \\
\hline $\begin{array}{l}\text { Information } \\
\text { sources }\end{array}$ & 9 & $\begin{array}{l}\text { Describe all intended information sources } \\
\text { (such as electronic databases, contact with } \\
\text { study authors, trial registers or other grey } \\
\text { literature sources) with planned dates of } \\
\text { coverage }\end{array}$ & $6-7$ \\
\hline Search strategy & 10 & $\begin{array}{l}\text { Present draft of search strategy to be used } \\
\text { for at least one electronic database, includ- } \\
\text { ing planned limits, such that it could be } \\
\text { repeated }\end{array}$ & Appendix 2 \\
\hline \multicolumn{4}{|l|}{ Study records: } \\
\hline $\begin{array}{l}\text { Data man- } \\
\text { agement }\end{array}$ & $11 \mathrm{a}$ & $\begin{array}{l}\text { Describe the mechanism(s) that will be } \\
\text { used to manage records and data through- } \\
\text { out the review }\end{array}$ & 10 \\
\hline $\begin{array}{l}\text { Selection } \\
\text { process }\end{array}$ & $11 b$ & $\begin{array}{l}\text { State the process that will be used for se- } \\
\text { lecting studies (such as two independent re- } \\
\text { viewers) through each phase of the review } \\
\text { (that is, screening, eligibility and inclusion } \\
\text { in meta-analysis) }\end{array}$ & 10 \\
\hline $\begin{array}{l}\text { Data } \\
\text { collection } \\
\text { process }\end{array}$ & $11 \mathrm{c}$ & $\begin{array}{l}\text { Describe planned method of extracting data } \\
\text { from reports (such as piloting forms, done } \\
\text { independently, in duplicate), any processes } \\
\text { for obtaining and confirming data from in- } \\
\text { vestigators }\end{array}$ & $10-11$ \\
\hline Data items & 12 & $\begin{array}{l}\text { List and define all variables for which data } \\
\text { will be sought (such as PICO items, fund- } \\
\text { ing sources), any pre-planned data assump- } \\
\text { tions and simplifications }\end{array}$ & $10-11$ \\
\hline $\begin{array}{l}\text { Outcomes and } \\
\text { prioritization }\end{array}$ & 13 & $\begin{array}{l}\text { List and define all outcomes for which data } \\
\text { will be sought, including prioritization of } \\
\text { main and additional outcomes, with ratio- } \\
\text { nale }\end{array}$ & 8 \\
\hline $\begin{array}{l}\text { Risk of bias } \\
\text { in individual } \\
\text { studies }\end{array}$ & 14 & $\begin{array}{l}\text { Describe anticipated methods for assessing } \\
\text { risk of bias of individual studies, including } \\
\text { whether this will be done at the outcome or } \\
\text { study level, or both; state how this informa- } \\
\text { tion will be used in data synthesis }\end{array}$ & 11 \\
\hline
\end{tabular}




\begin{tabular}{|c|c|c|c|}
\hline \multirow[t]{4}{*}{ Data synthesis } & $15 \mathrm{a}$ & $\begin{array}{l}\text { Describe criteria under which study data } \\
\text { will be quantitatively synthesised }\end{array}$ & $9-10$ \\
\hline & $15 b$ & $\begin{array}{l}\text { If data are appropriate for quantitative } \\
\text { synthesis, describe planned summary mea- } \\
\text { sures, methods of handling data and meth- } \\
\text { ods of combining data from studies, includ- } \\
\text { ing any planned exploration of consistency } \\
\text { (such as I }{ }^{2}, \text { Kendall's } \tau \text { ) }\end{array}$ & $12-14$ \\
\hline & $15 \mathrm{c}$ & $\begin{array}{l}\text { Describe any proposed additional analyses } \\
\text { (such as sensitivity or subgroup analyses, } \\
\text { meta-regression) }\end{array}$ & $12-14$ \\
\hline & $15 d$ & $\begin{array}{l}\text { If quantitative synthesis is not appropriate, } \\
\text { describe the type of summary planned }\end{array}$ & $13-14$ \\
\hline $\begin{array}{l}\text { Confidence in } \\
\text { cumulative evi- } \\
\text { dence }\end{array}$ & 17 & $\begin{array}{l}\text { Describe how the strength of the body } \\
\text { of evidence will be assessed (such as } \\
\text { GRADE) }\end{array}$ & 13 \\
\hline \multicolumn{4}{|c|}{$\begin{array}{l}\text { *It is strongly recommended that this checklist be read in conjunction with the PRISMA-P Explanation and Elaboration (cite when available) for import- } \\
\text { ant clarification on the items. Amendments to a review protocol should be tracked and dated. The copyright for PRISMA-P (including checklist) is held } \\
\text { by the PRISMA-P Group and is distributed under a Creative Commons Attribution Licence 4.0. }\end{array}$} \\
\hline \multicolumn{4}{|c|}{$\begin{array}{l}\text { From: Shamseer L, Moher D, Clarke M, Ghersi D, Liberati A, Petticrew M, Shekelle P, Stewart L, PRISMA-P Group. Preferred reporting items for systematic } \\
\text { review and meta-analysis protocols (PRISMA-P) 2015: elaboration and explanation. BMJ. } 2015 \text { Jan 2;349(jan02 1):g7647. }\end{array}$} \\
\hline
\end{tabular}

\section{Appendix 2. Search strategy in the databases.}

1) Database: Ovid MEDLINE(R) ALL <1946 to December 14, 2020>

Search Strategy:

1 sitting.mp. or Sitting Position/

2 "prolonged sitting".mp.

3 (prolong* adj3 (sedentar* or sit* or sitting)).mp.

4 ((uninterrupt* or excessive) adj2 (sit* or sedent*)).mp.

5 ((workplace or office) adj2 sit\$).mp.

6 ((workplace or office) adj2 seat $\$)$.mp.

7 (sedentar* adj2 (lifestyle or behavio?r)).mp.

8 (excessive adj2 (sit* or sedentar*)).mp.

9 (workplace adj2 (sedentar* or sit*)).mp.

10 (sedentar* adj2 (behavio?r or position or posture)).mp.

11 "sedentary behavio? r".mp. or exp *Sedentary Behavior/

121 or 2 or 3 or 4 or 5 or 6 or 7 or 8 or 9 or 10 or 11

13 (break* adj3 (sedentar* or sit*)).mp.

14 (interrupt* adj2 (prolong* or sedentary or sit*)).mp.

15 (walk* adj2 (activit* or bout*)).mp.

16 ((walk* or activit* or cycl* or exercis*) adj2 break*).mp.

17 (break* adj3 (up or sedentary or prolong* or sit* or behavio? r*)).mp.

18 ((interrupt* or disrupt* or replac*) adj3 (sedentar* or sit* or "prolonged sitting")).mp.

19 (desk adj3 (office or job or work)).mp.

20 microbreak*.mp. 
21 (movement adj2 break*).mp.

2213 or 14 or 15 or 16 or 17 or 18 or 20 or 21

2312 or 19

24 (inflammat* adj2 (marker* or risk*)).mp.

25 (acute adj2 protein).mp.

26 ((Inflammat* or biochemi*) adj3 marker*).mp.

27 (chronic adj3 (inflammat* or "systemic inflammat*" or "low grade inflammation")).mp.

28 (immun* adj2 (reaction or response*)).mp.

29 (interleukin or IL-6 or IL-8).mp.

30 (C adj3 (reactive protein* or RP)).mp.

31 C-Reactive Protein/

32 *Adipokines/

33 (Tumo?r adj2 necrosis).mp.

34 Tumor Necrosis Factor-alpha.mp. or exp *Tumor Necrosis Factor-alpha/

3524 or 25 or 26 or 27 or 28 or 29 or 30 or 31 or 32 or 34

3623 and 35 [For objective one]

3722 and 23 and 35 [For objective two]

2) Database: Scopus <Inception to December 17, 2020>

\section{Objective 1}

( ( ( ( TITLE-ABS-KEY ( sitting ) OR TITLE-ABS-KEY ( «sitting position») OR TITLE-ABS-KEY ( «sedentary position») OR TITLE-ABS-KEY ( «prolonged sitting»)) ) OR ( ( TITLE-ABS-KEY ( «excessive sitting») OR TITLE-ABS-KEY ( «prolonged sitting») OR TITLE-ABS-KEY ( «uninterrupted sitting»)) ) OR ( ( TITLE-ABS-KEY ( sedentar*) OR TITLE-ABS-KEY ( sit**) OR TITLE-ABS-KEY ( «uninterrupted sitting») )) OR (( TITLE-ABS-KEY ( lifestyle) OR TITLE-ABS-KEY ( «lifestyle behavio? $r »)))$ OR ( ( TITLE-ABS$\mathrm{KEY}($ «workplacesitting») ORTITLE-ABS-KEY(«officesitting») ORTITLE-ABS-KEY( «workplaceseat"») ORTITLE-ABSKEY ( «desk-based»))) OR ( TITLE-ABS-KEY (prolong* PRE/3 ( sedentar* OR sit* OR sitting)) ) OR ( TITLEABS-KEY (uninterrupt*PRE/2 (sit*OR sedent*)) ) OR ((TITLE-ABS-KEY ( workplace OR office) PRE/2 sit\$) OR TITLEABS-KEY ( ( workplace OR office) PRE/2 seat\$)) ) OR ( ( TITLE-ABS-KEY ( excessive PRE/2 ( sit* OR sedentar $\left.\left.^{*}\right)\right)$ OR TITLE-ABS-KEY (sedentar*PRE/2 (lifestyle OR behavio? r) )) ) OR ((TITLE-ABS-KEY (workplace PRE/2 (sedentar*OR sit*)) ORTITLE-ABS-KEY (sedentar*PRE/2 (behavio?rORposition ORposture))) ) OR((TITLE-ABS-KEY( «sedentary behavio?r» "sedentary AND behavior) OR TITLE-ABS-KEY ( «"Sedentary Behavior»)))) OR ( TITLE-ABSKEY (deskPRE/2 (office OR jobOR work)) )) AND ((TITLE-ABS-KEY (inflammat*PRE/2 (marker*OR risk*))) OR (TITLEABS-KEY (acute PRE/2 protein)) OR (TITLE-ABS-KEY ( (inflammat* OR biochemi*) PRE/2 marker*) ) OR (TITLE-ABSKEY ( inflammat*)) OR ( TITLE-ABS-KEY ( cbronic PRE/2 ( inflammat" OR «systemic inflammat"» OR «low grade inflammation»))) ) OR ( TITLE-ABS-KEY (chronic PRE/2 (inflammat* OR «systemic inflammat"» OR «low grade inflammation»)) ) OR ( ( TITLE-ABS-KEY ( cytokine) OR TITLE-ABS-KEY ( immun* PRE/2 (reaction OR response*) )) ) OR ( (TITLE-ABS-KEY (interleukin OR il-6 OR il-8) OR TITLE-ABS-KEY ( «C Reactive Protein») OR TITLE-ABS-KEY ( «C-Reactive protein»))) OR ( TITLE-ABS-KEY ( crp ) ) OR ( TITLE-ABSKEY ( "adipokine) ) OR ( TITLE-ABS-KEY ( tumo?r PRE/2 necrosis )) OR ( ( TITLE-ABS-KEY ( «Tumor Necrosis Factor-alpha») OR TITLE-ABS-KEY ( tnf*) OR TITLE-ABS-KEY ( «"Tumor Necrosis Factor-alpha») ) ) ) AND (LIMITTO ( LANGUAGE, «English») ) AND ( LIMIT-TO ( SRCTYPE, «j»)) AND ( EXCLUDE ( EXACTKEYWORD, «Nonbuman») OR EXCLUDE (EXACTKEYWORD, «Animals»)) AND (LIMIT-TO (DOCTYPE, «ar»))

\section{Objective 2}

( ( ( ( TITLE-ABS-KEY ( sitting ) OR TITLE-ABS-KEY ( «sitting position») OR TITLE-ABS-KEY ( «sedentary position») OR TITLE-ABS-KEY ( «prolonged sitting»)) ) OR ( ( TITLE-ABS-KEY ( «excessive sitting») OR TITLE-ABS-KEY ( «prolonged sitting») OR TITLE-ABS-KEY ( «uninterrupted 
sitting») )) OR ( ( TITLE-ABS-KEY ( sedentar*) OR TITLE-ABS-KEY ( sit*) OR TITLE-ABS-KEY ( «uninterrupted sitting») )) OR ( ( TITLE-ABS-KEY ( lifestyle) OR TITLE-ABS-KEY ( lifestyle bebavio? $r \gg)))$ OR ( ( TITLE-ABSKEY («workplacesitting») ORTITLE-ABS-KEY («officesitting») ORTITLE-ABS-KEY( «workplaceseat"») ORTITLE-ABSKEY ( «desk-based»))) OR ( TITLE-ABS-KEY (prolong* PRE/3 ( sedentar* OR sit* OR sitting)) ) OR ( TITLEABS-KEY (uninterrupt*PRE/2 (sit*OR sedent*) )) OR ((TITLE-ABS-KEY ( (workplace OR office) PRE/2 sit\$) OR TITLEABS-KEY ( ( workplace OR office) PRE/2 seat\$))) OR ( ( TITLE-ABS-KEY ( excessive PRE/2 ( sit* OR sedentar $\left.\left.^{*}\right)\right)$ OR TITLE-ABS-KEY (sedentar*PRE/2 (lifestyle OR behavio? $\left.\left.r\right)\right)$ )) OR ((TITLE-ABS-KEY (workplace PRE/2 (sedentar*ORsit*)) ORTITLE-ABS-KEY(sedentar*PRE/2 (bebavio?rORpositionORposture)))) OR((TITLE-ABS-KEY( «sedentary behavio?r» "sedentary AND behavior) OR TITLE-ABS-KEY ( «"Sedentary Behavior»)))) OR ( TITLE-ABSKEY (deskPRE/2 (office ORjob OR work $)))$ ) AND ((TITLE-ABS-KEY (inflammat*PRE/2 (marker*OR risk*)) ) OR (TITLEABS-KEY (acute PRE/2 protein) ) OR (TITLE-ABS-KEY ( (inflammat* OR biochemi*) PRE/2 marker*)) OR (TITLE-ABSKEY ( inflammat*) ) OR ( TITLE-ABS-KEY ( chronic PRE/2 ( inflammat* OR «systemic inflammat"» OR «low grade inflammation»)) ) OR ( TITLE-ABS-KEY ( cbronic PRE/2 ( inflammat* OR «systemic inflammat"» OR «low grade inflammation»)) ) OR ( ( TITLE-ABS-KEY (cytokine) OR TITLE-ABS-KEY ( immun* PRE/2 (reaction OR response*)) )) OR ( ( TITLE-ABS-KEY ( interleukin OR il-6 OR il-8) OR TITLE-ABS-KEY ( «C Reactive Protein») OR TITLE-ABS-KEY ( «C-Reactive protein»))) OR ( TITLE-ABS-KEY ( crp ) ) OR ( TITLEABS-KEY ( "adipokine) ) OR ( TITLE-ABS-KEY ( tumo?r PRE/2 necrosis)) OR ( ( TITLE-ABS-KEY ( «Tumor Necrosis Factor-alpha») OR TITLE-ABS-KEY ( $t n f^{*}$ ) OR TITLE-ABS-KEY ( "*Tumor Necrosis Factor-alpha»)) ) ) AND ( ( ( TITLE-ABS-KEY ( break* PRE/2 ( sedentar* OR sit*)) OR TITLE-ABS-KEY ( ( interrupt* OR disrupt*) PRE/2 ( prolong* OR sedentary OR sit*) ) OR TITLE-ABS-KEY ( ( reallocat* OR replac*) PRE/2 (prolong* OR sedentary OR sit*)))) OR ( ( TITLE-ABS-KEY ( walk* PRE/2 (activ$i t^{*}$ OR bout*)) OR TITLE-ABS-KEY ( ( walk* OR activit* OR cycl* OR exercis*) PRE/2 break*) OR TITLEABS-KEY ( break* PRE/2 ( up OR sedentary OR prolong* OR sit* OR bebavio? $\left.r^{*}\right)$ )) ) OR (( TITLEABS-KEY ( ( interrupt* OR disrupt* OR replac*) PRE/3 ( sedentar* OR sit* OR «prolonged sitting») ) OR TITLE-ABS-KEY (microbreak* ) OR TITLE-ABS-KEY ( movement PRE/2 break*)))) AND ( LIMIT-TO ( LANGUAGE, «English»)) AND ( EXCLUDE ( EXACTKEYWORD, «Nonbuman») OR EXCLUDE (EXACTKEYWORD, «Animals»))

3) Database: CINAHL <Inception to December 18, 2020>

$\begin{array}{ll}\# & \text { Query } \\ \text { S14 } & \text { (S6 AND S9 AND S13) } \\ \text { S13 } & \text { (S6 AND S12) } \\ \text { S12 } & \text { (S10 OR S11) } \\ \text { S11 } & \text { "Tumor Necrosis Factor-alpha" OR *Tumor Necrosis Factor-alpha } \\ & \text { ( inflammat* N2 (marker* or risk*) ) OR acute N2 phase N2 protein OR ( (Inflammat* or } \\ & \text { biochemi*) N3 marker*) OR ( chronic N2 (inflammat* or "systemic inflammat*" or "low } \\ & \text { grade inflammation") ) OR ( immun* N2 (reaction or response*) ) OR ( interleukin* or IL-6 } \\ & \text { or IL-8 ) OR "plasminogen activator" OR leptin OR ( "c-reactive protein*) or crp OR "C } \\ & \text { reactive protein") OR *Adipokines OR "low grade inflammation" OR ( "Tumo?r N2 necro- } \\ \text { S10 } & \text { sis N2 factor) OR TNF ) }\end{array}$

S9 (S7 OR S8)

( (Cycl* OR exercis* OR danc* OR walk* OR calisthenic*) adj2 (activit* or bout*)) OR ( (walk* or activit* or cycl* or exercis*) N2 break*) OR ( (break* N3 (up or sedentary or prolong* or sit* or behavio? r*) ) OR ( (interrupt* or disrupt* or replac*) N3 (sedentar* or sit* or "prolonged sitting")) OR micro* N2 break OR microbreak* OR ( movement N2 (break or interrupt*) ) 
( break* N3 (sedentar* or sit*) ) OR ( (interruptions or distraction or disruption) N2 (sit* OR seat* or sedentar*) ) OR ( (interrupt* N2 (prolong* or sedentary or sit*) ) OR ( (replac*

S7

S6

S5

S4

S3

S2

S1
OR reallocat*) N2 (prolong* or sedentary or sit*))

(S1 OR S2 OR S3 OR S4 OR S5)

desk N3 (office or job or work)

( sedentar* N2 (behavio?r or position or posture) ) OR ( "sedentary behavio? r" OR *Sedentary Behavior/ ) OR ( sedentary lifestyle or sedentary behavior or inactivity ) OR ( physical inactivity or physically inactive or sedentary )

( desk* N2 (based OR bound) N2 (office OR job or Work*) ) OR ( sedentar* N2 (lifestyle or behavio?r) ) OR ( excessive N2 (sit* or sedentar*) ) OR ( workplace N2 (sedentar* or sit*) )

( (uninterrupt* or excessive) N2 (sit* or sedent*) ) OR ( (workplace or office) N2 sit\$ ) OR ( (workplace or office) $\mathrm{N} 2$ seat $\$$ )

( sitting OR "Sitting Position" ) OR "prolonged sitting" OR ( prolong* N2 (sedentar* or sit* or sitting) 